\title{
LEP Search for Single Top production and new fermions
}

Mario Antonelli

LNF-INFN

Frascati

ICHEP 2002

Amsterdam

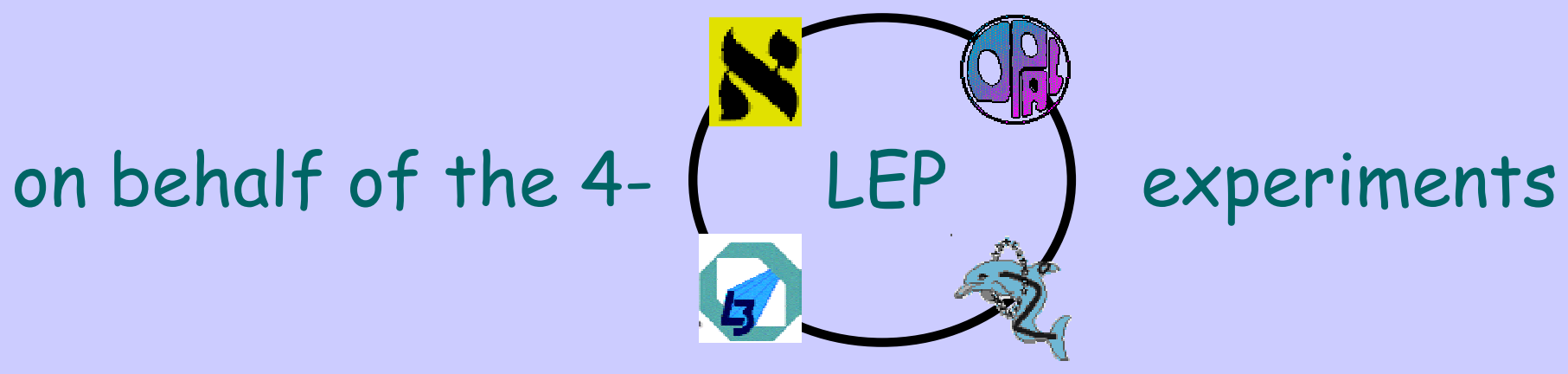




\section{The LEP $e^{+} e^{-}$collider}

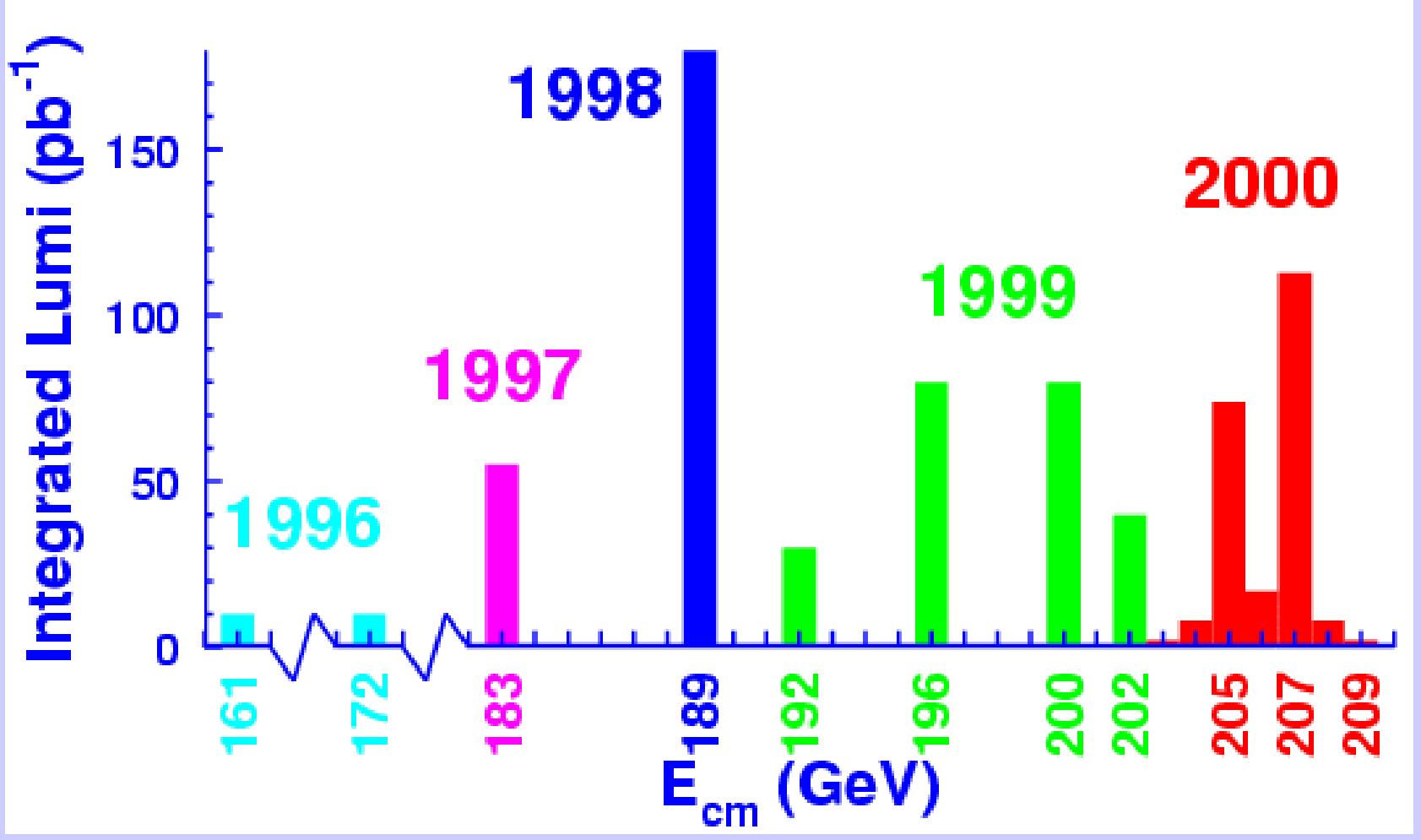

High centre of mass energy + Luminosity + clean experimental environment

All limits are at $95 \% C L$ and preliminary 
Search for single top production via FCNC

$$
e^{+} e^{-} \rightarrow+\bar{q}(\bar{q}=\bar{c}, \bar{u})
$$

At tree level SM, there are no vertices for these FCNC processes (GIM mechanism $\mathrm{V}_{C K M}=\mathrm{V}_{C K M}{ }^{-1}$ ).

FCNC appear at loop level
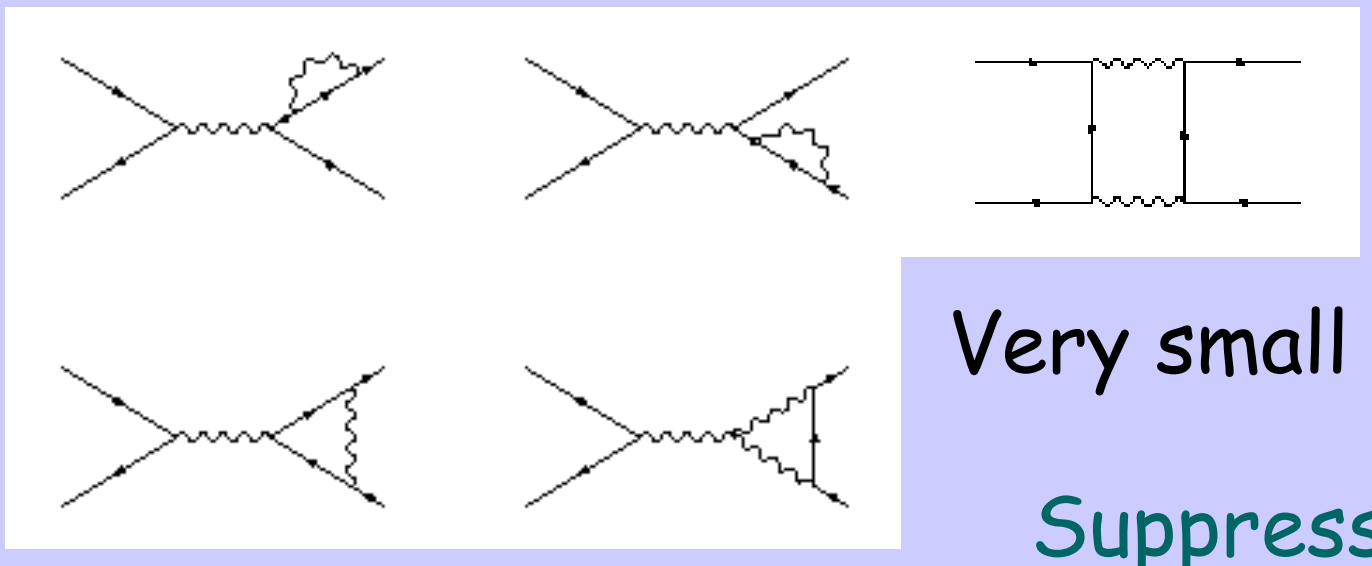

Very small cross section ( $10^{-9} \mathrm{fb}$ )

Suppressed by loop + GIM

Extensions of SM could lead to enhancement of such transitions 
The FCNC vertices tqV $(V=\gamma, Z)$ probed in

- rare decays of top quark

- top-q associated production

\section{Existing limits}

TEVATRON: $\mathrm{p} \overline{\mathrm{p}} \rightarrow+\bar{\dagger} \mathrm{X}$

\section{CDF:}

$\mathrm{BR}(\dagger \rightarrow \mathrm{c} \gamma)+\mathrm{BR}(\dagger \rightarrow \mathrm{u} \gamma)<3.2 \%$ $\mathrm{BR}(\dagger \rightarrow \mathrm{CZ})+\mathrm{BR}(\dagger \rightarrow \mathrm{uZ})<33 \%$

$\rightarrow$ very weak constraint: $\mathrm{N}_{\mathrm{Ht}} \sim 10^{2}$ HERA: ep $\rightarrow$ eutX

Sensitivity to tur coupling $\mathrm{K}_{\text {utr }}$ ZEUS:

$B R(t \rightarrow u \gamma)<0.7 \%\left(K_{\text {tur }}<0.19\right)$ $\sigma$ corresponding to CDF limits

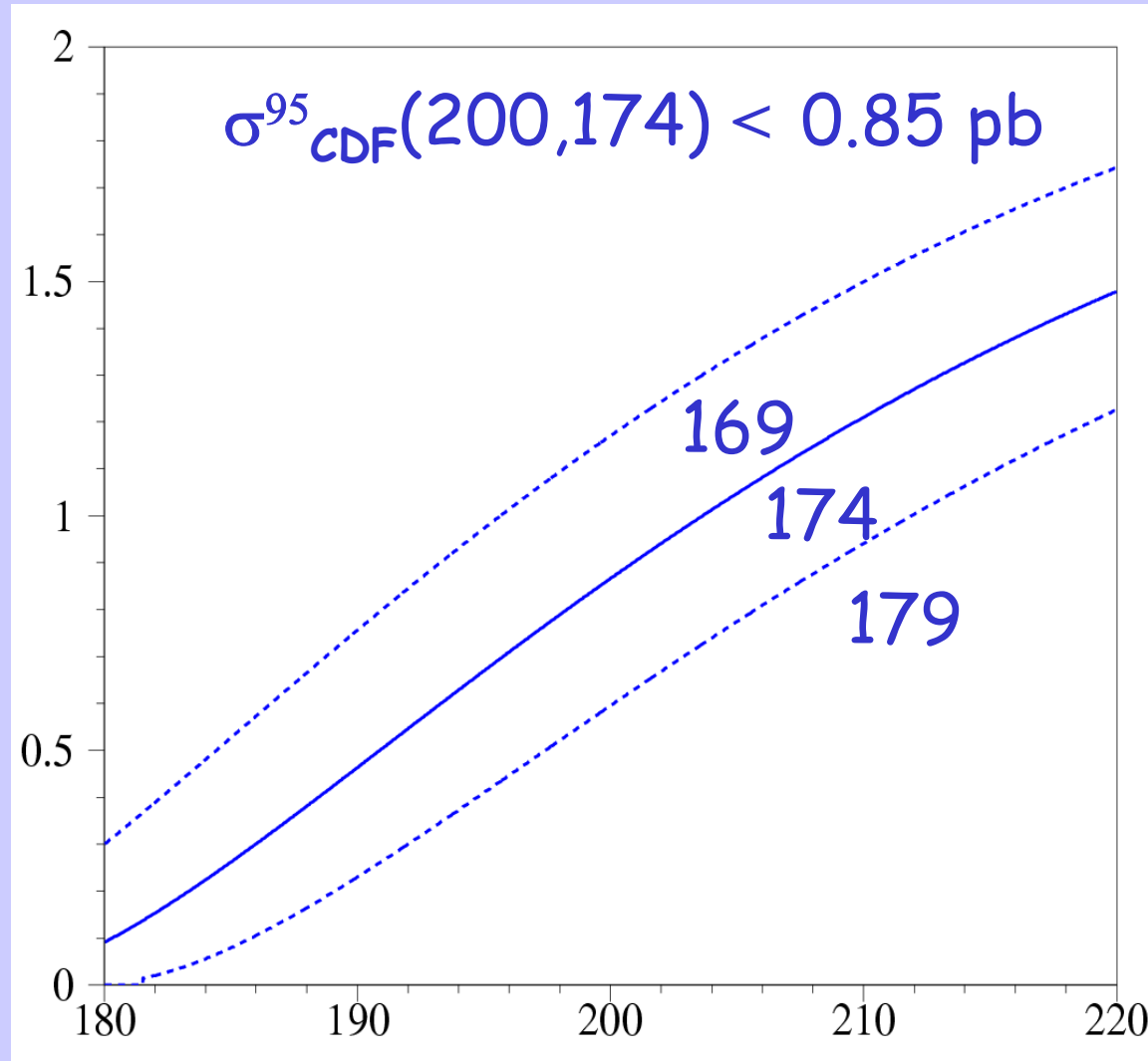




\section{Topologies}

Top decays rapidly via

$\left(10^{-24} \mathrm{sec}\right) \Longrightarrow$ no top hadrons formed

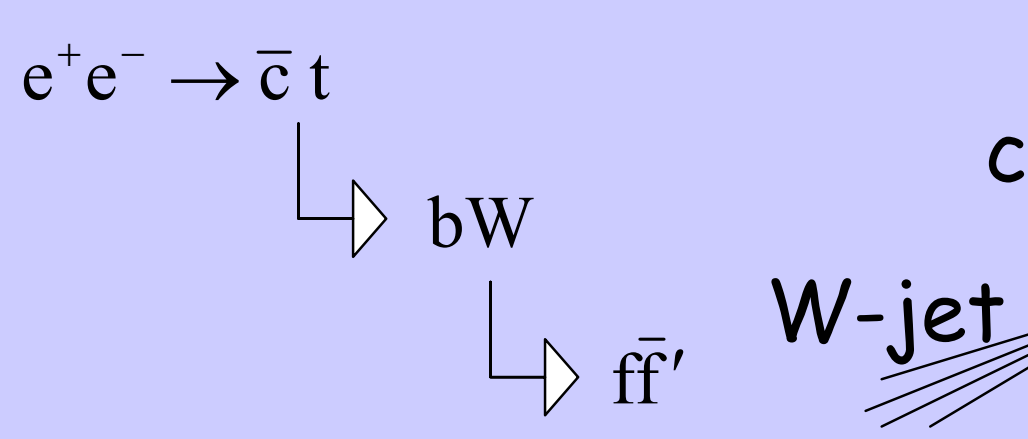

Kinematics at $189 \mathrm{GeV}$

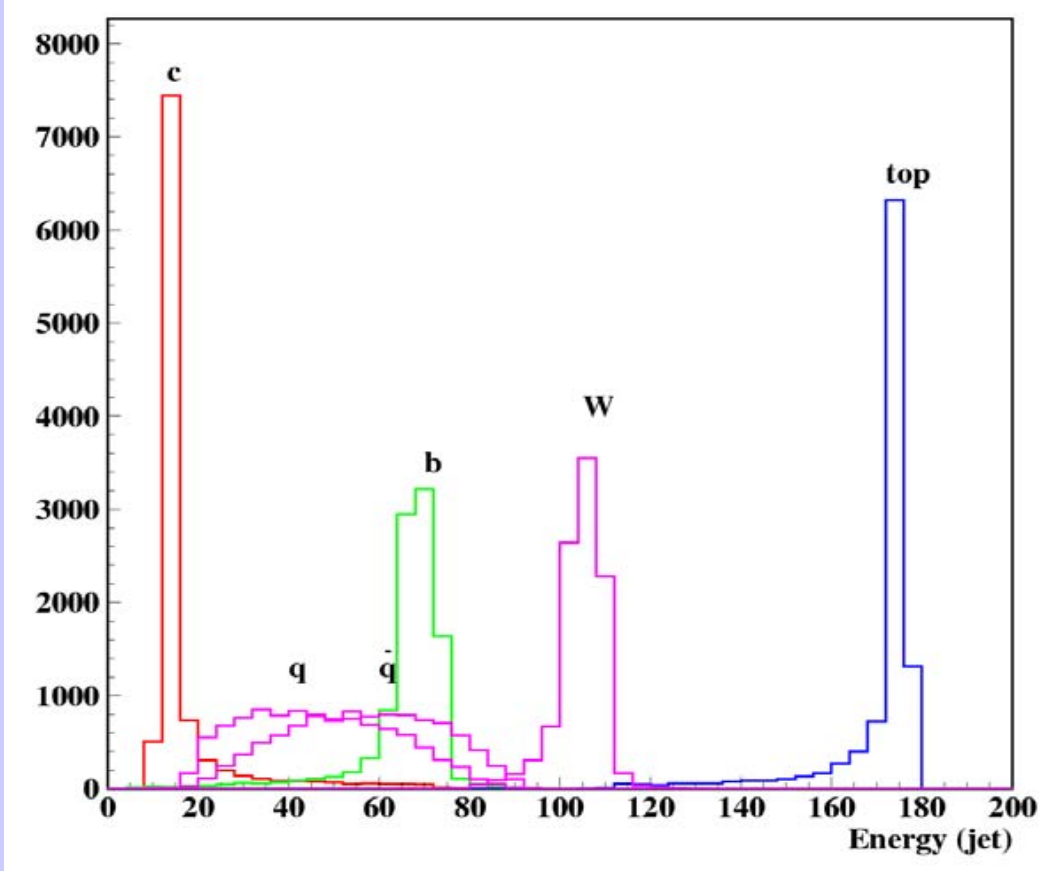

b-jet

b-jet

$E_{\dagger} \sim m_{+}$

$E_{W} \sim\left(m_{+}^{2}+m_{W}^{2}-m_{b}^{2}\right) / 2 m_{+}$

$E_{b} \sim\left(m_{t}^{2}-m_{W^{2}}+m_{b}^{2}\right) / 2 m_{t}$

$E_{q} \sim \sqrt{s}-m_{+}$

W-jet

$\ell$ 


\section{Selections and performances}

2 selections: hadronic kinematics + b-tag

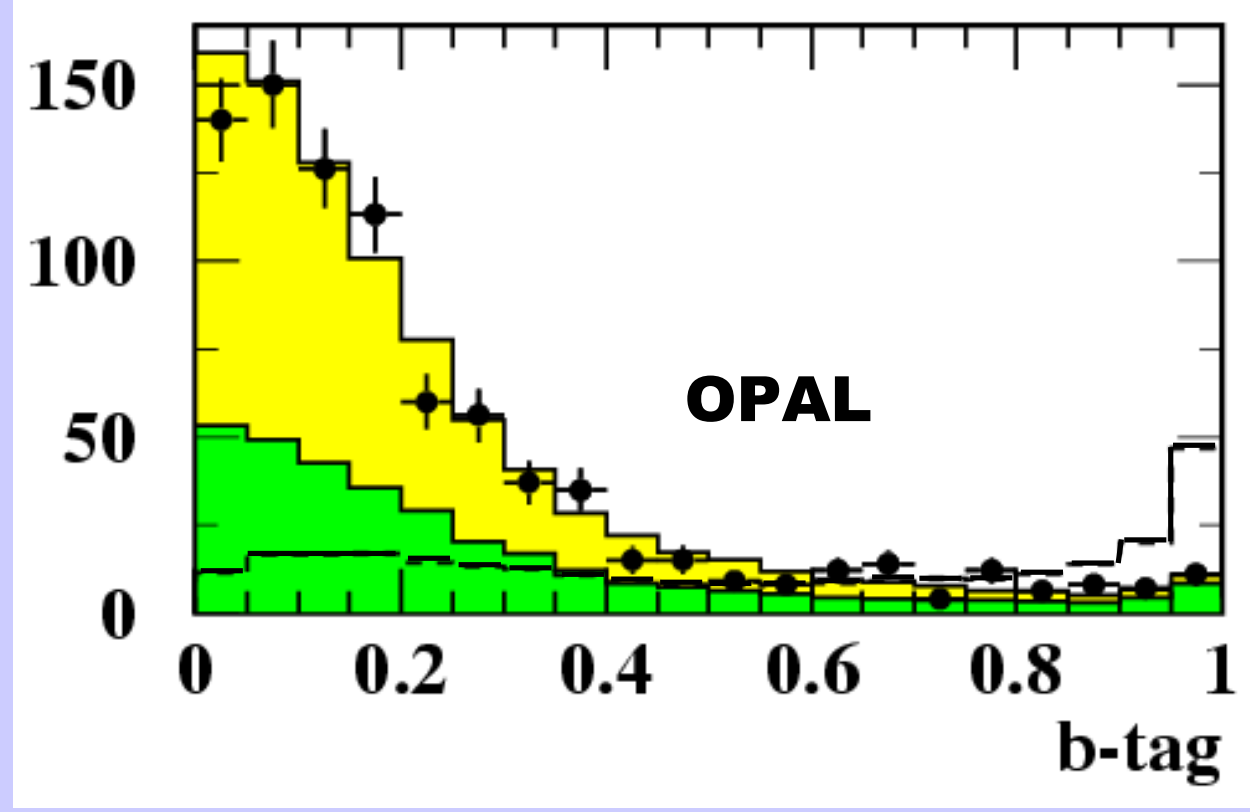

$\varepsilon \sim 10 \%-15 \%$

$B k g \sim 50-200 \mathrm{fb}(W W, 99)$ and Leptonic $W$ decay

+ lepton id + Emiss

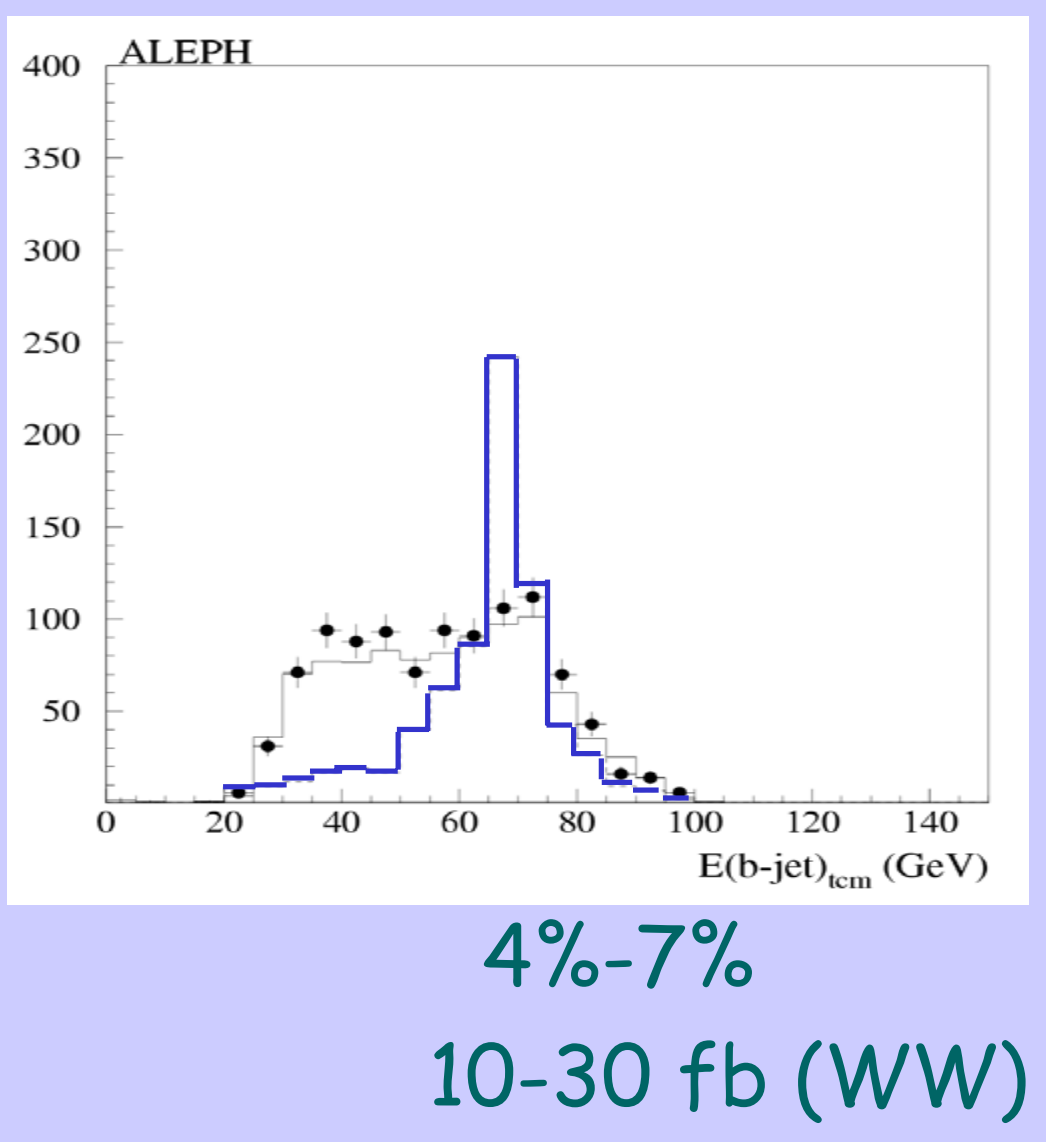

No excess seen in data 


\section{Combined* LEP results LEPEX TICA WG}

Cross section upper limits for $m_{+}=174 \mathrm{GeV}$

\begin{tabular}{|c|c|c|}
\hline $\begin{array}{c}<\sqrt{\mathrm{s}>} \\
\mathrm{GeV}\end{array}$ & $\begin{array}{c}\sigma 95(\mathrm{obs}) \\
\mathrm{pb}\end{array}$ & $\begin{array}{c}\sigma 95(\exp ) \\
\mathrm{pb}\end{array}$ \\
\hline 189 & 0.11 & 0.11 \\
\hline 192 & 0.38 & 0.33 \\
\hline 196 & 0.36 & 0.20 \\
\hline 200 & 0.21 & 0.21 \\
\hline 202 & 0.30 & 0.35 \\
\hline 205 & 0.20 & 0.25 \\
\hline 207 & 0.17 & 0.18 \\
\hline
\end{tabular}

About $0.55 \mathrm{fb}^{-1}$ per experiment * LR method for combination
Limits on FCNC couplings

LEP Preliminary

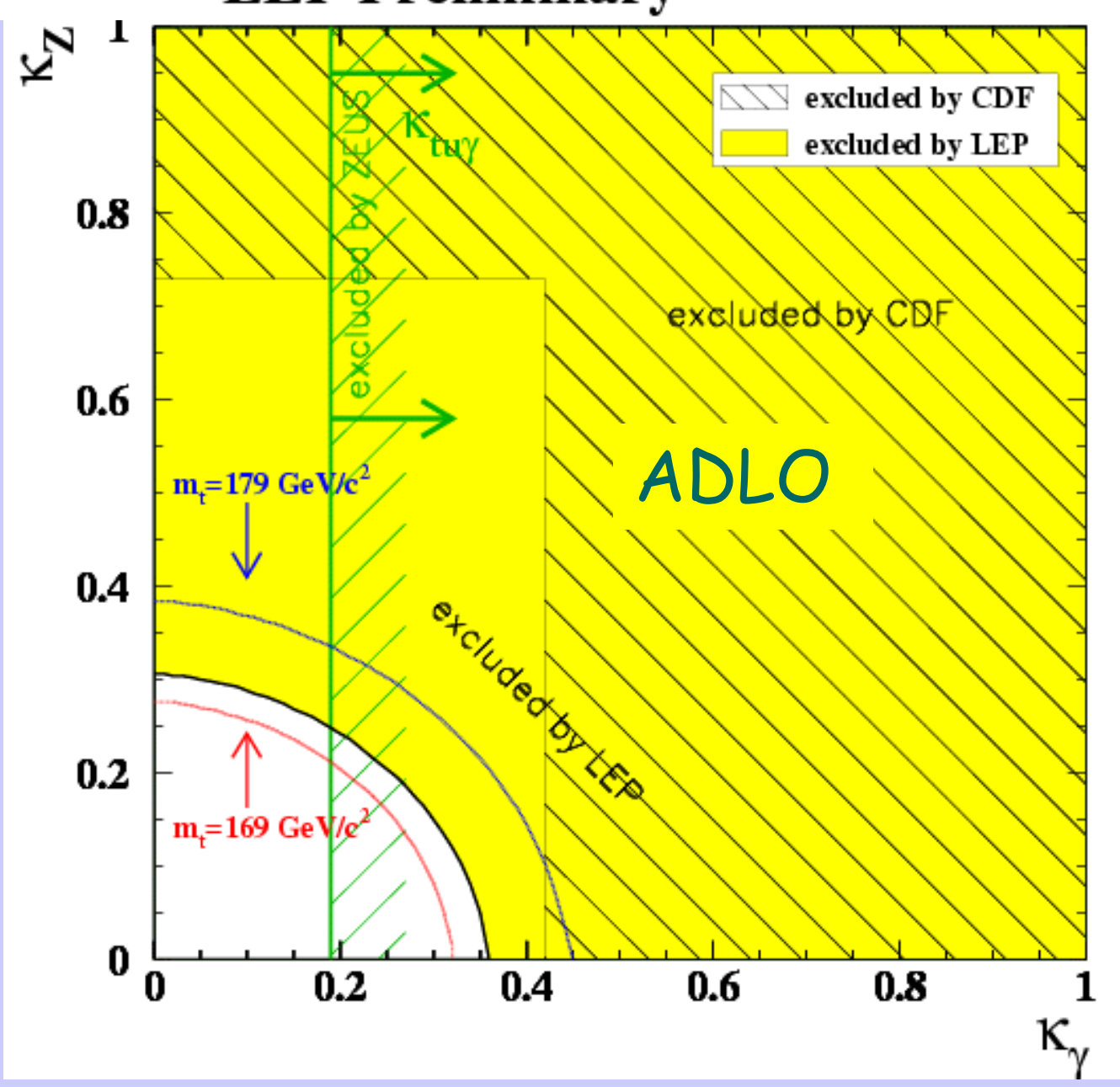




\section{Combined* LEP results LEPEX TICA WG}

Cross section upper limits for $m_{t}=174 \mathrm{GeV}$

\begin{tabular}{|c|c|c|}
\hline $\begin{array}{c}<\sqrt{\mathrm{s}}> \\
\mathrm{GeV}\end{array}$ & $\begin{array}{c}\sigma 95(\mathrm{obs}) \\
\mathrm{pb}\end{array}$ & $\begin{array}{c}\sigma 95(\exp ) \\
\mathrm{pb}\end{array}$ \\
\hline 189 & 0.11 & 0.11 \\
\hline 192 & 0.38 & 0.33 \\
\hline 196 & 0.36 & 0.20 \\
\hline 200 & 0.21 & 0.21 \\
\hline 202 & 0.30 & 0.35 \\
\hline 205 & 0.20 & 0.25 \\
\hline 207 & 0.17 & 0.18 \\
\hline
\end{tabular}

About $0.55 \mathrm{fb}^{-1}$ per experiment

*LR method for combination
Limits on branching ratios

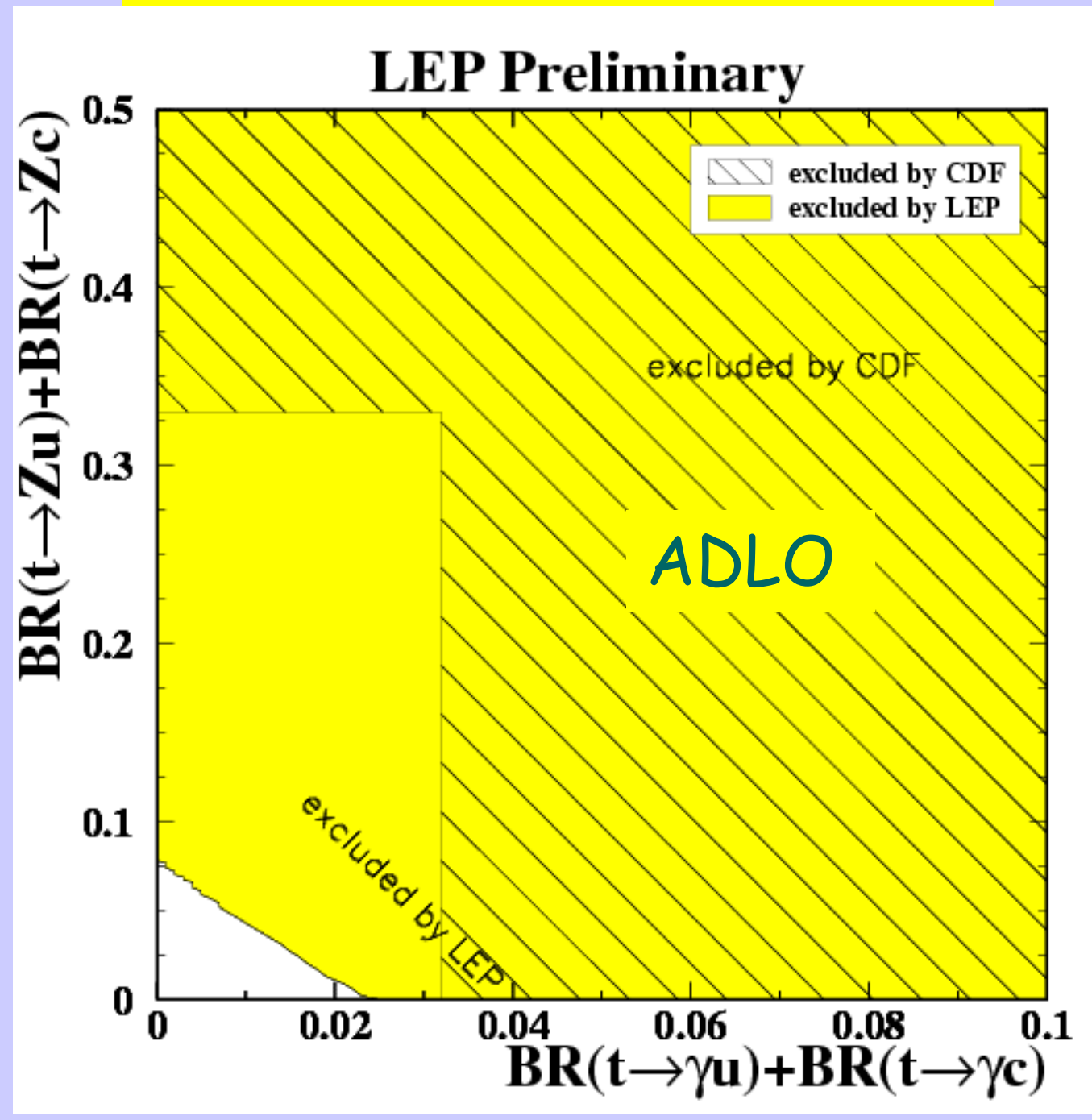




\section{Search for excited and heavy fermions}

Fermions in SM

and beyond
$\left(\begin{array}{l}v_{e} \\ e^{-}\end{array}\right)_{L}\left[\begin{array}{l}v_{\mu} \\ \mu^{-}\end{array}\right)_{L}\left(\begin{array}{l}v_{\tau} \\ \tau^{-}\end{array}\right)_{L}$
New fundamental particles?
Sequential, vector, mirror
$\mathrm{L}^{ \pm} \mathrm{L}^{0}$
$\mathrm{e}_{\mathrm{R}}^{-}$
$\mu_{\mathrm{R}}^{-}$
$\tau_{\mathrm{R}}^{-}$
Isosinglet neutrino
$\mathrm{N}_{1}$
$\left(\begin{array}{l}u \\ d\end{array}\right)_{L}\left[\begin{array}{l}c \\ s\end{array}\right)_{L}\left(\begin{array}{l}t \\ b\end{array}\right)_{L}$
Heavy quarks
$b^{\prime}$

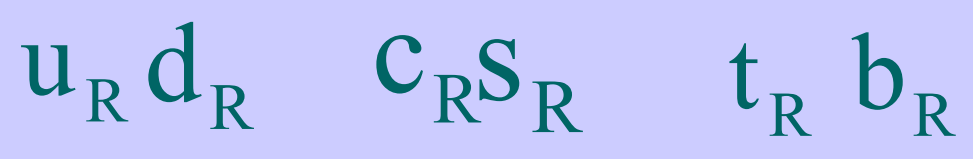
Excited Leptons
$1^{*} v_{1}^{*}$ 


\section{Search for heavy fermions}

Production:
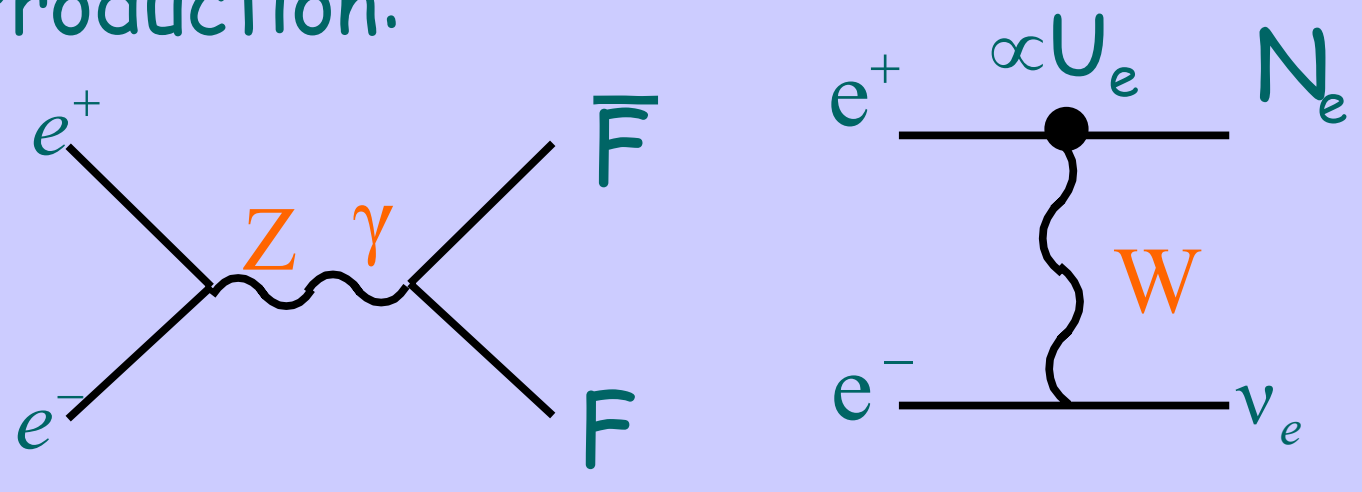

Decay*:
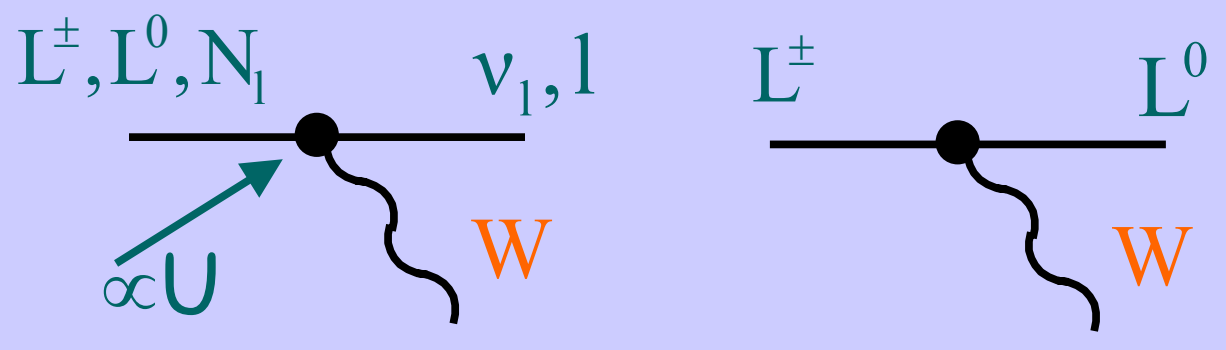

* decay to $W$ favored these energies

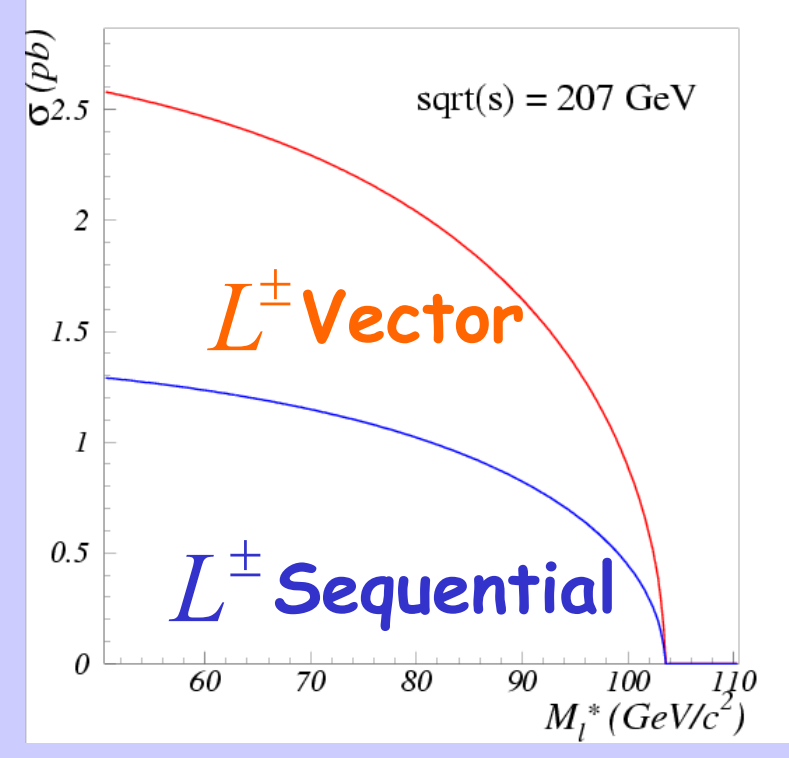

NEW

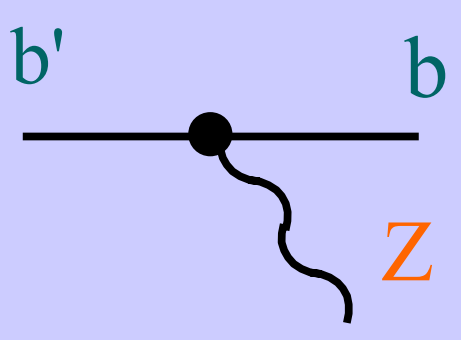

Could be sizeable if :

$$
\mathrm{M}_{\mathrm{b}^{\prime}}<\mathrm{M}_{\mathrm{t}}\left(\mathrm{M}_{\mathrm{t}^{\prime}}\right)+\mathrm{M}_{\mathrm{W}}
$$




\section{Heavy leptons}

\section{Jets and leptons}

$L^{0} \rightarrow l W$

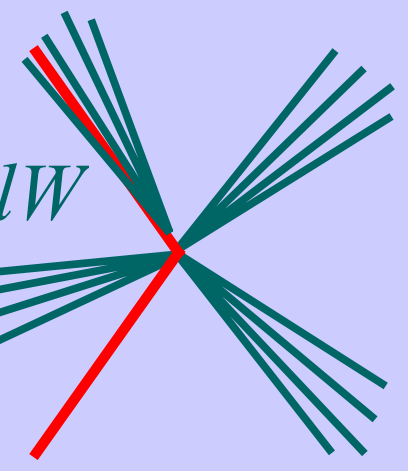

Heavy stable charged particles

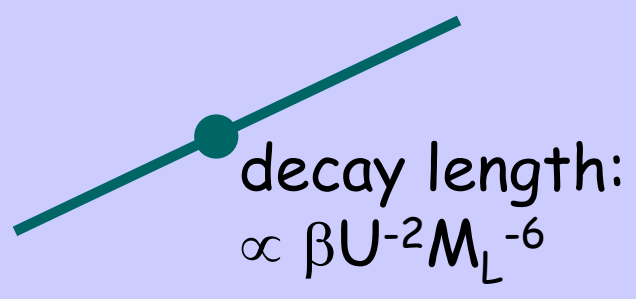

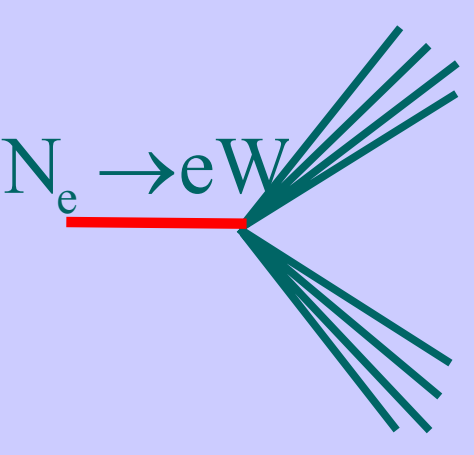

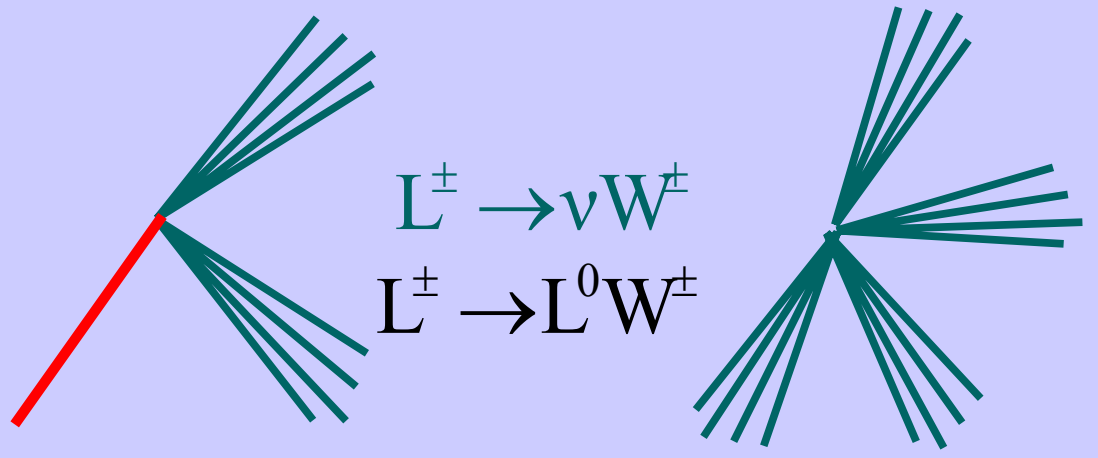

$E_{\text {miss }}+P_{\text {tmiss }}$ depending on $M_{L^{ \pm}}-M_{L^{0}}$

small $U$ and $\mathrm{M}_{\mathrm{L}^{ \pm}}<\mathrm{M}_{\mathrm{L}^{0}}$

$$
\text { J-L HSCP }
$$

$$
\begin{aligned}
\mathcal{E} & \sim 35-15 \%(5 \%) & & 65 \% \\
\mathrm{Bkg}^{*} & \sim 0.3-1 \mathrm{pb} & & 0.01 \mathrm{pb}
\end{aligned}
$$

Candidates in agreement with SM expectation ${ }^{\star} W W, f \bar{f}(\gamma), Z Z, \gamma \gamma, \ldots$ 


\section{Lower mass limits}

\begin{tabular}{|c|c|c|c|c|c|}
\hline Decay Mode & Model & Dirac & Majorana & Decay Mode & \\
\hline $\mathrm{L}^{0} \rightarrow \mathrm{e} W$ & $\begin{array}{l}\text { Sequential } \\
\text { Vector } \\
\text { Mirror }\end{array}$ & $\begin{array}{l}101.3 \\
102.6 \\
100.8\end{array}$ & $\begin{array}{l}89.5 \\
---- \\
89.5 \\
\end{array}$ & $\mathrm{~L}^{ \pm} \rightarrow v \mathrm{~W}$ & $\begin{array}{l}100.8 \\
101.2 \\
100.5\end{array}$ \\
\hline $\mathrm{L}^{0} \rightarrow \mu \mathrm{W}$ & $\begin{array}{l}\text { Sequential } \\
\text { Vector } \\
\text { Mirror }\end{array}$ & $\begin{array}{l}101.5 \\
102.7 \\
101.0\end{array}$ & $\begin{array}{l}90.7 \\
---- \\
90.7\end{array}$ & $\mathrm{~L}^{ \pm} \rightarrow \mathrm{L}^{0} \mathrm{~W}$ & $\begin{array}{l}101.9 \\
102.1 \\
101.9\end{array}$ \\
\hline $\mathrm{L}^{0} \rightarrow \tau \mathrm{W}$ & $\begin{array}{l}\text { Sequential } \\
\text { Vector } \\
\text { Mirror }\end{array}$ & $\begin{array}{l}90.3 \\
99.3 \\
90.3\end{array}$ & $\begin{array}{l}80.5 \\
---- \\
80.5\end{array}$ & Stable & $\begin{array}{l}102.6 \\
102.6 \\
102.6\end{array}$ \\
\hline
\end{tabular}




\section{Search for $e^{+} e^{-} \rightarrow b^{\prime} \bar{b}^{\prime} \rightarrow b \bar{b} Z Z$ NEW}
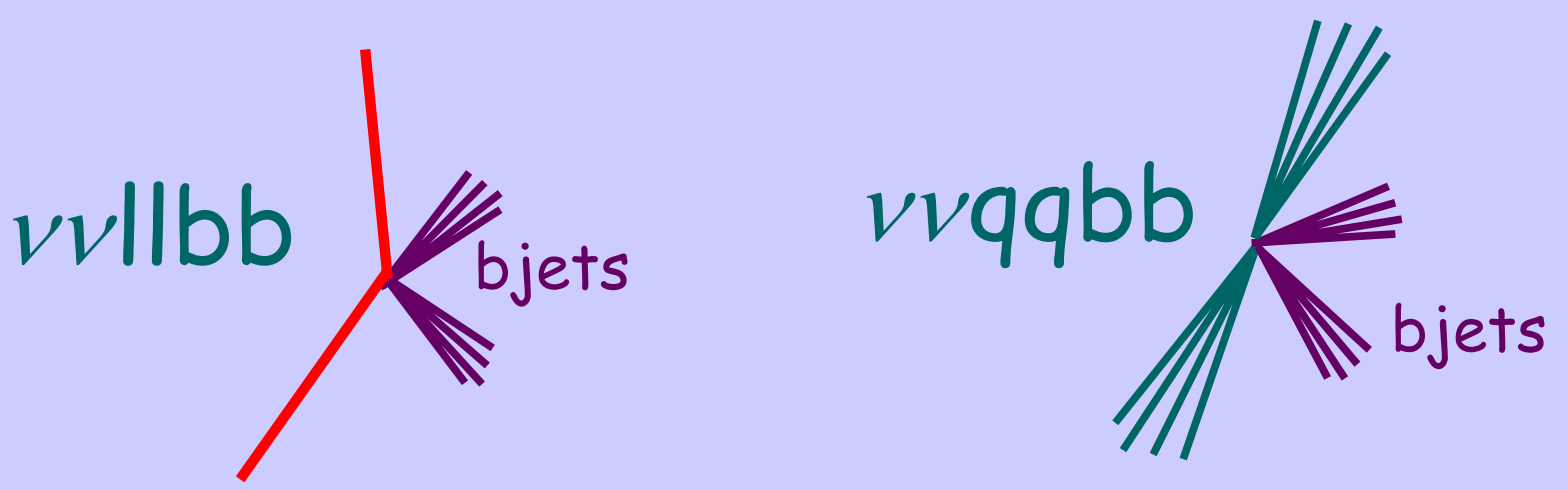

+ clean signature

- BR (ZZ->|lvv) 4\%

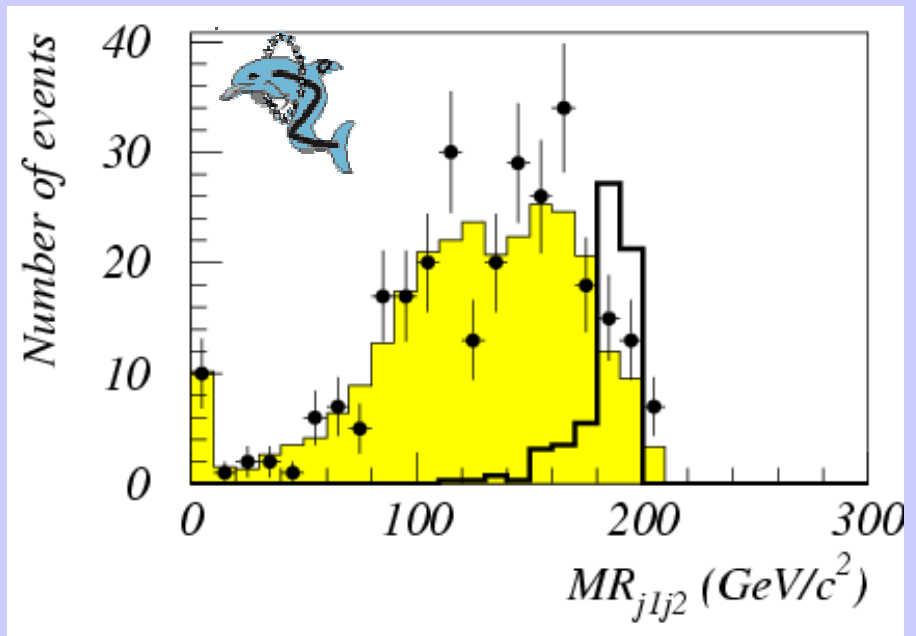

simple cut analysis
- large background

+ $B R(Z Z$->qqvv) 28\%

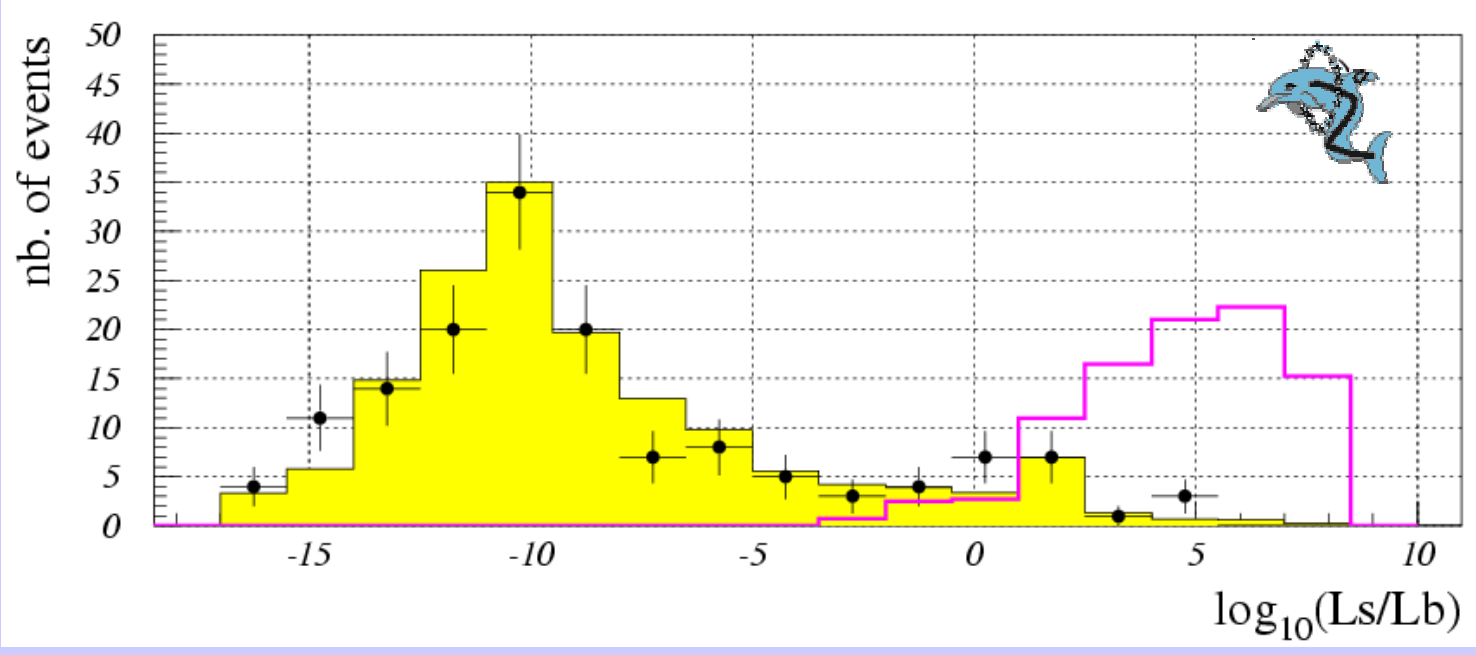

shape analysis 


\section{Search for $e^{+} e^{-} \rightarrow b^{\prime} \bar{b}^{\prime} \rightarrow b \bar{b} Z Z$ NEW}

\section{Preliminary results}

about $344 \mathrm{pb}^{-1}$ at energies above $200 \mathrm{GeV}$

\section{$v v$ llbb}

efficiency $\quad 250 \%(x 4 \%)$

background $\sim 8$

data

$\sigma 95(\exp )$

$\sigma 95$
8
8

$0.8 \mathrm{pb}$

$0.65 \mathrm{pb}$ $v v q q b b$

$\sim 40 \%(\times 28 \%)$ for $M_{b^{\prime}}=100 \mathrm{GeV}$ $\sim 665$

599

$0.29 \mathrm{pb}$

$0.26 \mathrm{pb}$

$\sigma 95=95 \% C L$ upper limit on $\sigma\left(e^{+} e^{-} \rightarrow b^{\prime} \bar{b}^{\prime}\right) B R\left(b^{\prime} \rightarrow b Z\right)^{2}$

Combined: $\sigma\left(e^{+} e^{-} \rightarrow b^{\prime} \bar{b}^{\prime}\right) B R\left(b^{\prime} \rightarrow b Z\right)^{2}<0.21 \mathrm{pb}$ for $M_{b^{\prime}}=100 \mathrm{GeV}$ $\sigma\left(e^{+} e^{-} \rightarrow b^{\prime} \bar{b}^{\prime}\right) S M \approx 0.6 \mathrm{pb}$ at $\sqrt{ } s=206.6 \mathrm{GeV}$

CDF limits (PRL 84 (2000) 835) $M_{b^{\prime}}=100 \mathrm{GeV}$ :

$\sigma\left(p \bar{p} \rightarrow b^{\prime} \bar{b}^{\prime}\right) B R\left(b^{\prime} \rightarrow b Z\right)^{2}<37 p b$

$\sigma\left(p \bar{p} \rightarrow b^{\prime} \bar{b}^{\prime}\right) S M \approx 102 p b$
DELPHI

$\mathrm{BR}\left(\mathrm{b}^{\prime} \rightarrow \mathrm{bZ}\right)^{2}<0.35$

CDF

$\mathrm{BR}\left(\mathrm{b}^{\prime} \rightarrow \mathrm{bZ}\right)^{2}<0.36$ 


\section{Search for excited leptons}

Substructure at a scale $\Lambda \Longrightarrow$ excited leptons

Prompt decay: $\ell^{*} v_{v_{1}^{*}}{ }_{\mathrm{f}_{\mathrm{v}} / \Lambda} v_{\gamma} \mathrm{v}_{1}$

$f, f^{\prime}$ control strength of $S U(2), U(1)$ couplings

(eg: $f=f^{\prime}$ no $v_{e}^{*} \rightarrow v_{e} \gamma f=-f^{\prime}$ no $e^{*} \rightarrow e \gamma$ )

Pair production

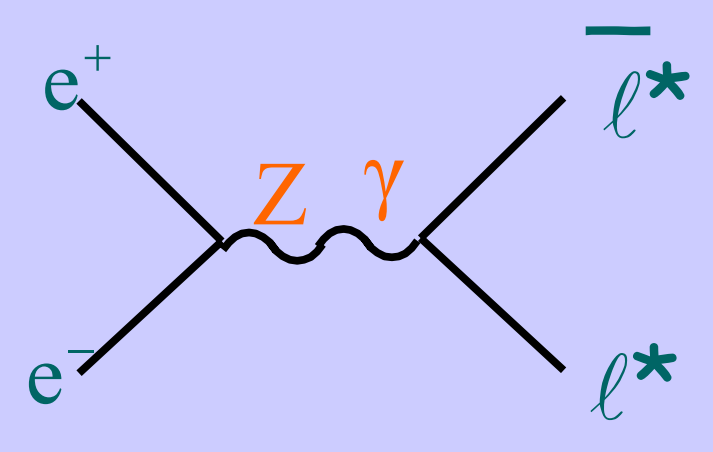

single production

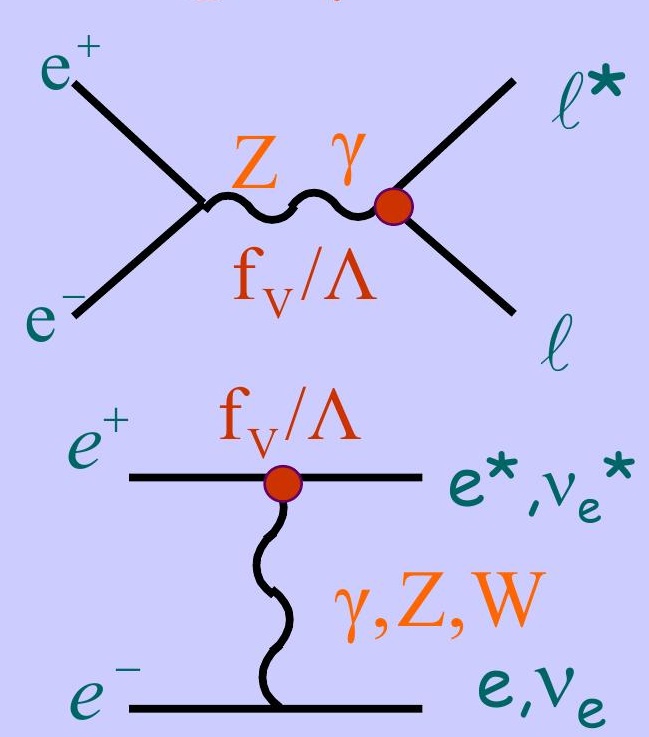

Virtual contribution

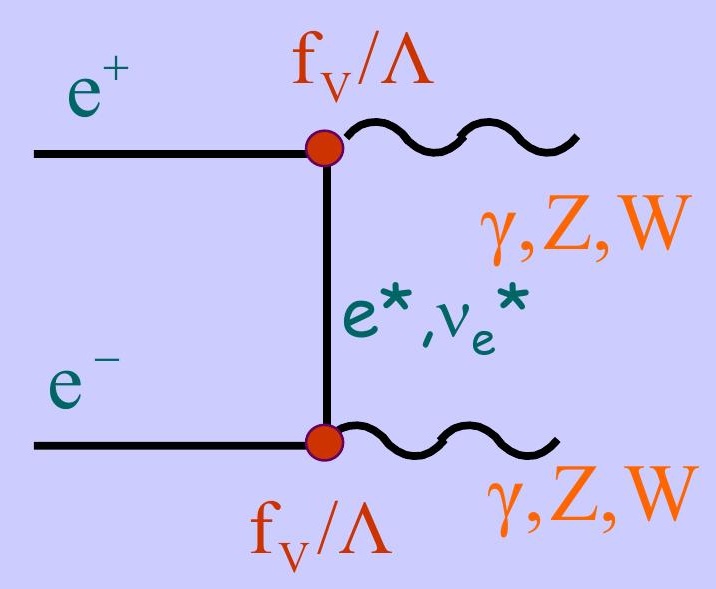




\section{Search for pair produced excited leptons}

Mass reach up to $\sqrt{ } / 2$

Topologies: as heavy leptons +

Photons, Leptons and photons

\begin{tabular}{|l|c|c|c|c|c|c|}
\hline & $\mathrm{e}^{*}$ & $\mu^{*}$ & $\tau^{*}$ & $v_{\mathrm{e}}^{*}$ & $\mathrm{~V}_{\mu}^{*}$ & $v_{\tau}^{*}$ \\
\hline \hline $\mathrm{f}=\mathrm{f}^{\prime}$ & 103.0 & 103.1 & 102.2 & 102.0 & 102.4 & 95.3 \\
\hline $\mathrm{f}=-\mathrm{f}^{*}$ & 98.0 & 98.0 & 98.0 & 102.7 & 102.8 & 102.8 \\
\hline
\end{tabular}

\begin{tabular}{|l|l|l|l|}
\hline $\mathrm{f}=\mathrm{f}^{\prime}$ & 102.9 & 102.9 & 102.8 \\
\hline
\end{tabular} 


\section{Single production}

Mass reach extended up to $\sim \sqrt{s}$

Many topologies:

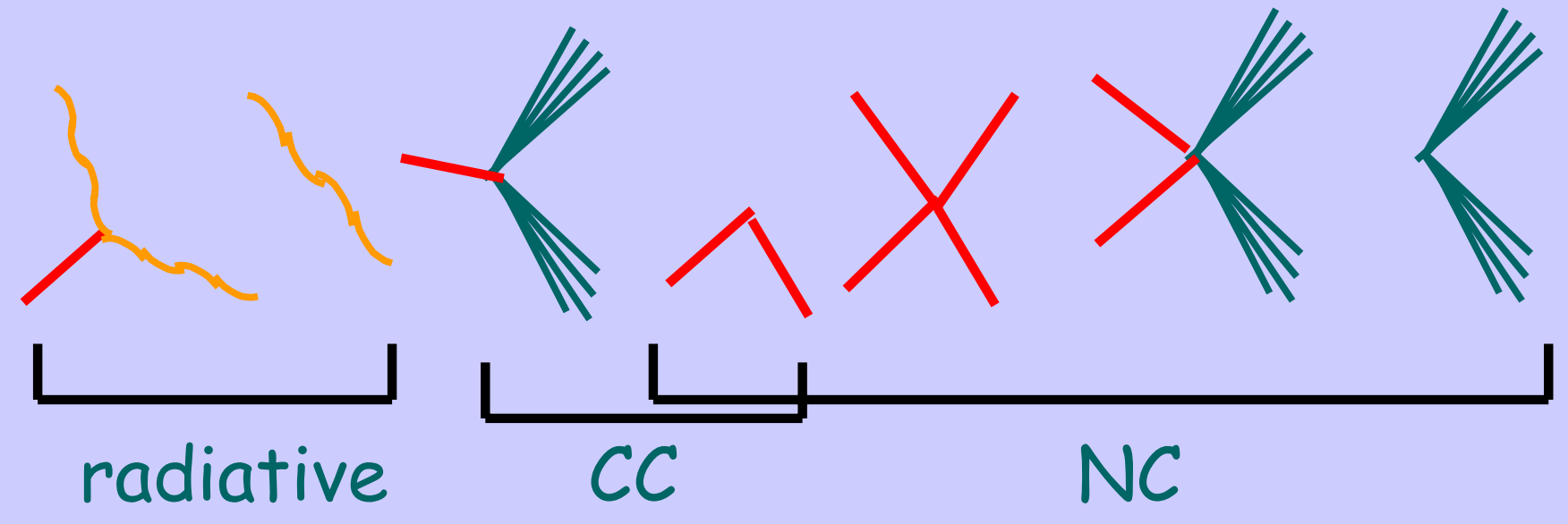

Typical efficiency: 15\% ( $\tau W)-60 \%(\mu \gamma)$ 


\section{Combined LEP results LEPEX TICA WG}

\section{$(1 / \mathrm{TeV})$}
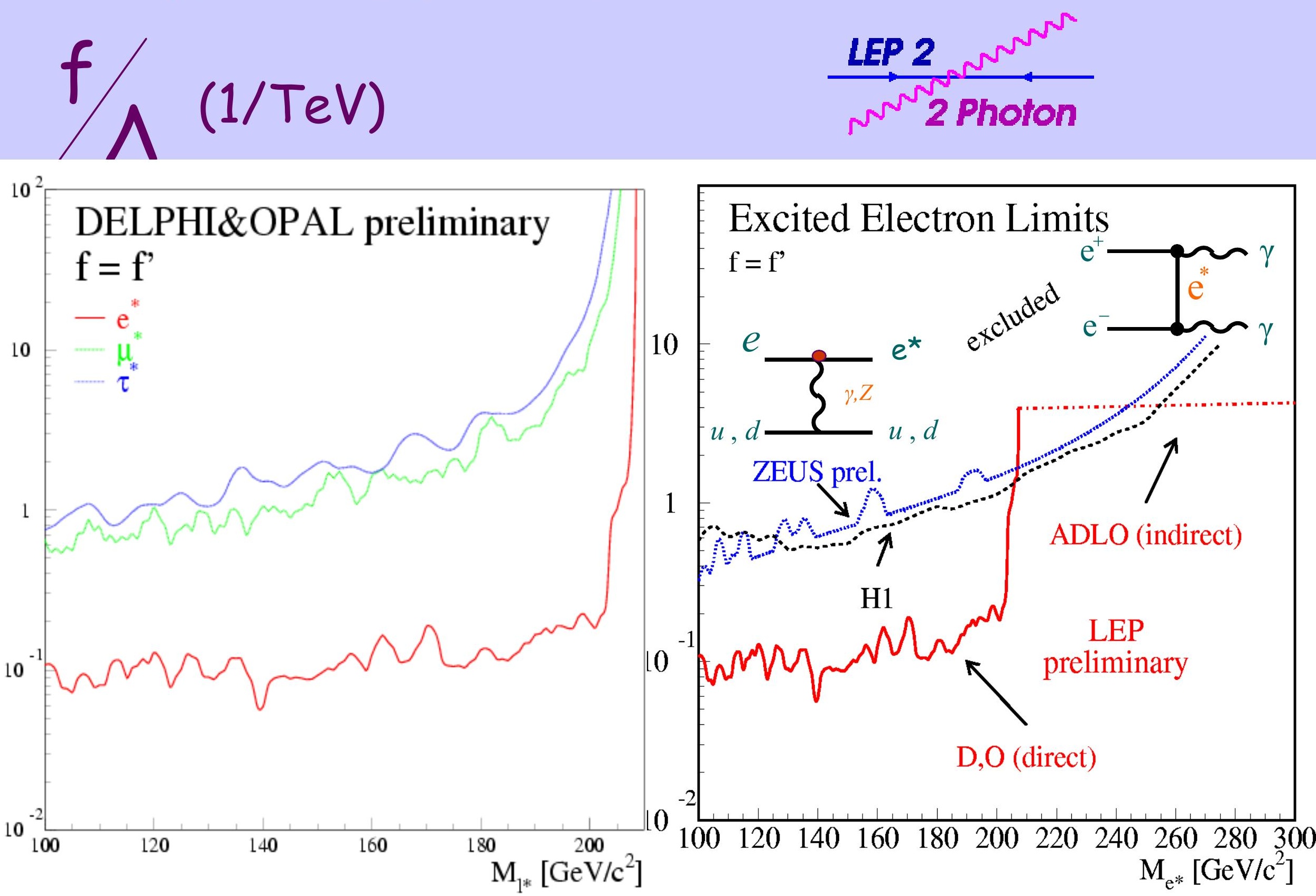


\section{Conclusion}

LEP is (was) beautifull:

centre-of-mass energies and luminosities above the specifications.

New physics thoroughly searched for with the $2.5 \mathrm{fb}^{-1}$ luminosity collected at LEP at

$E_{c m}$ up to $209 \mathrm{GeV}$

Numbers and properties of the events selected generally in agreement with SM expectation

Experimental constrains are dramatically improved.

We look forward to the near(?) future 


\section{$\mathrm{L}^{ \pm} \rightarrow v \mathrm{~W}^{ \pm}$}

4 jets and missing energy
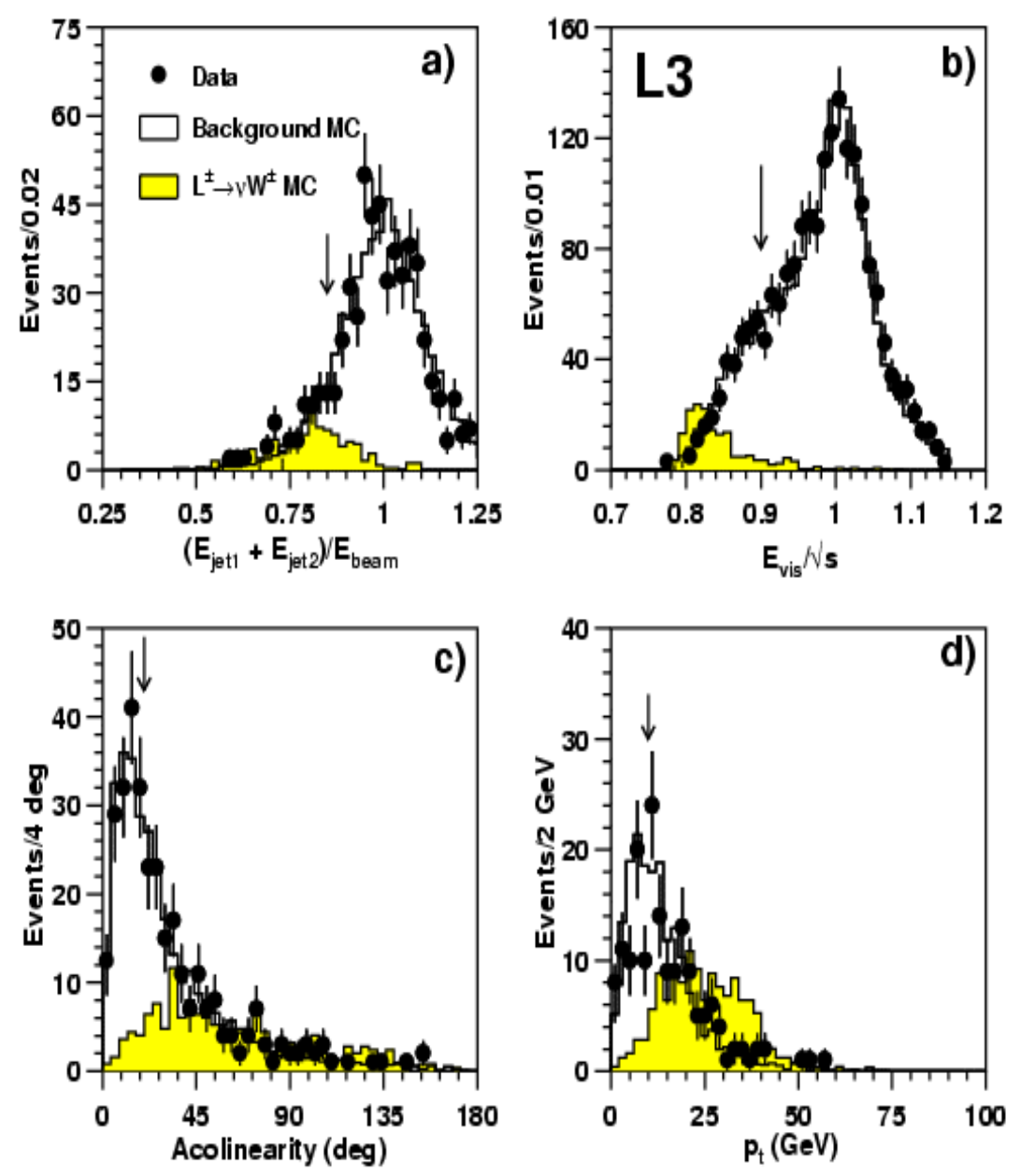

\section{$\mathrm{L}^{ \pm}$stable}

Anomalous ionization loss

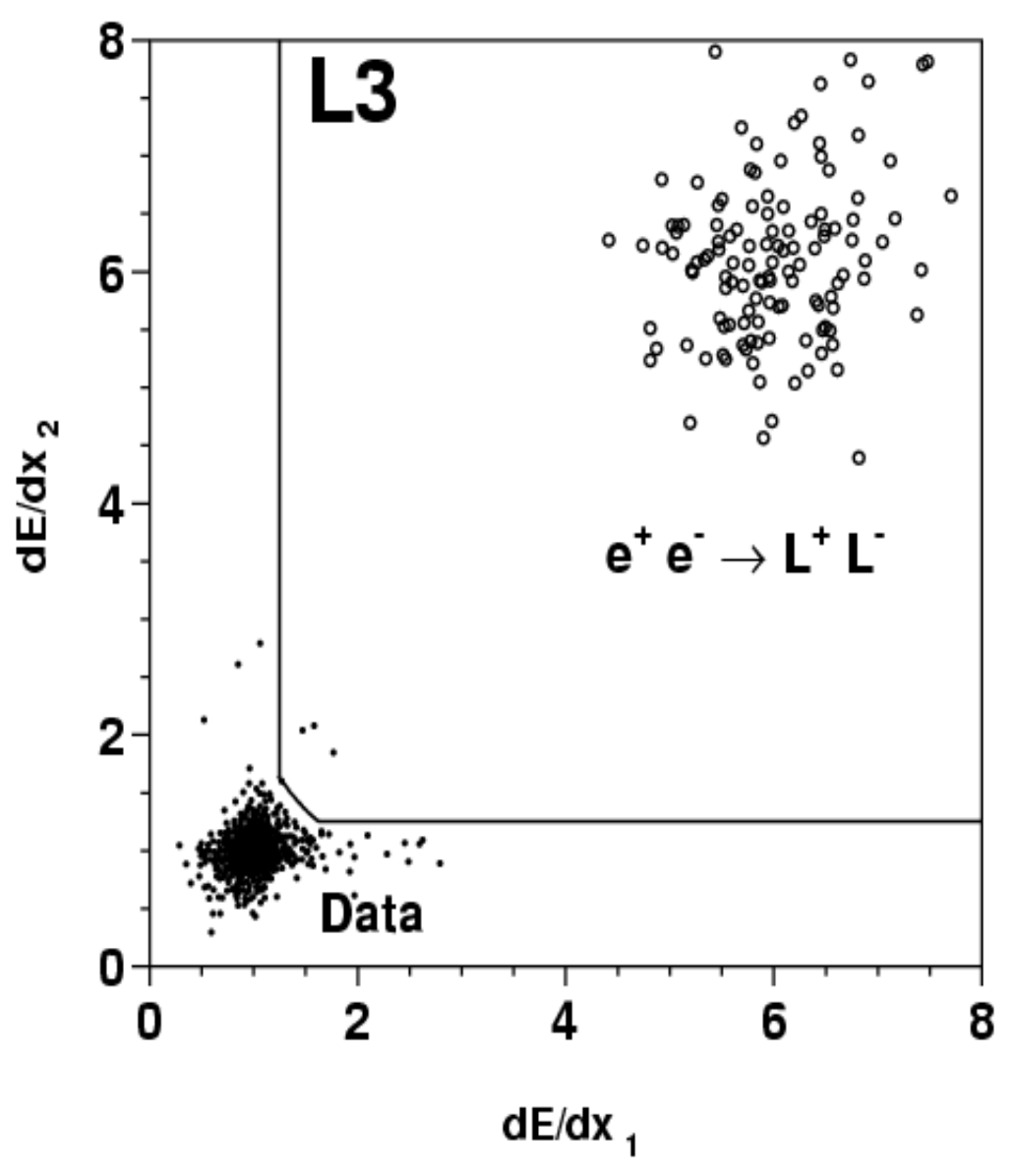




\section{Combined LEP results

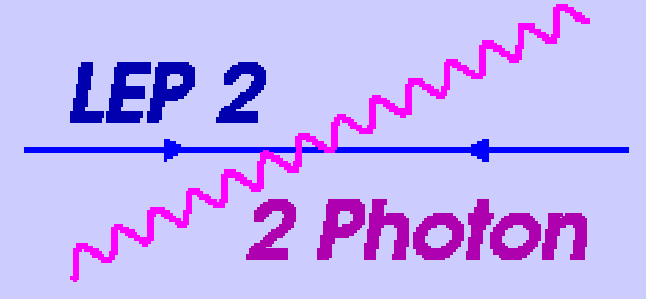

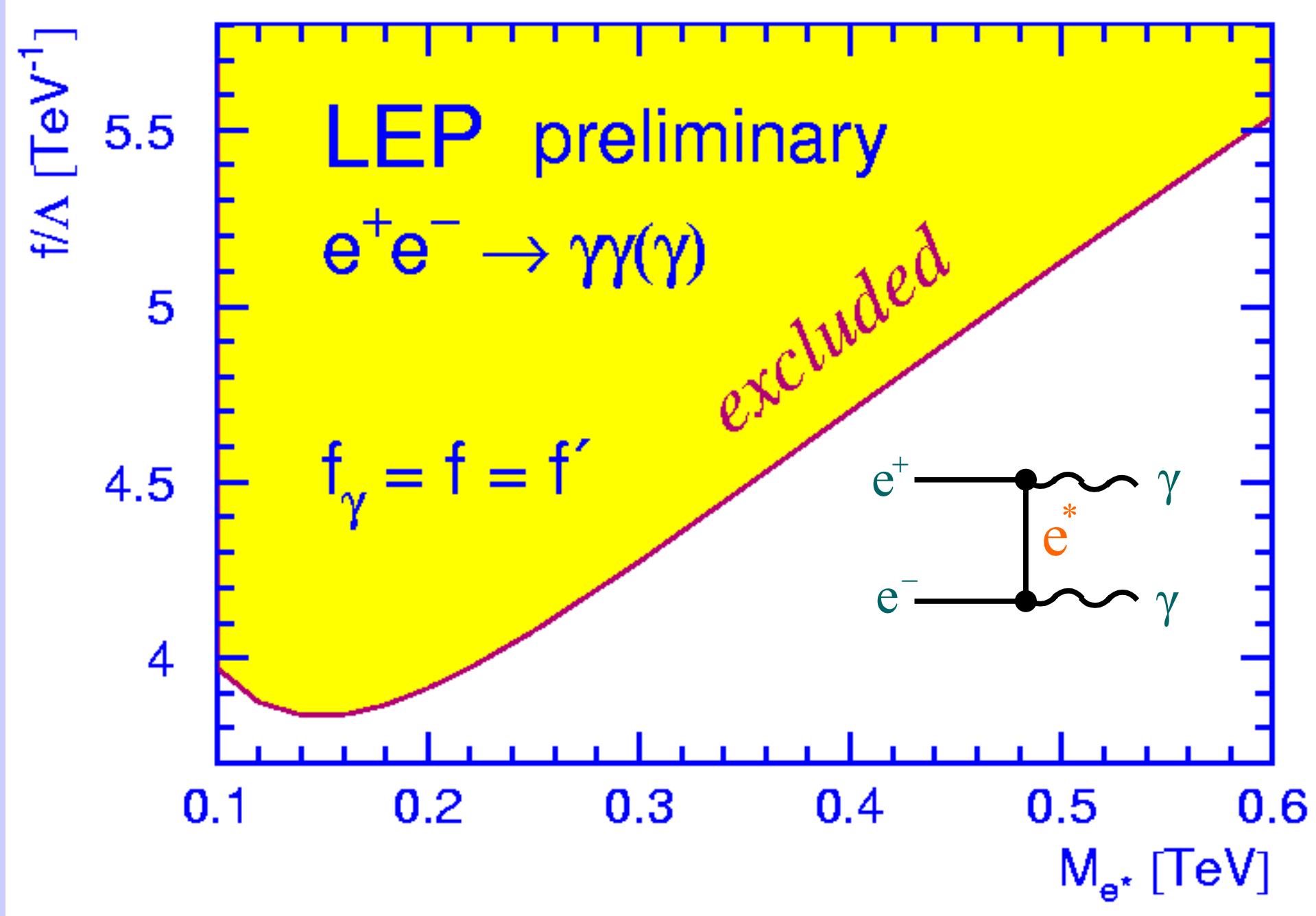

